\title{
Role of Cardiovascular Comorbidity and Depressive Symptoms on One-Year Clinical Progression of Alzheimer's Disease (AD) in a Population of Italian Elderly
}

\author{
Servello $A^{1^{*}}$, Artini $M^{1}$, Cerra $E^{2}$, Vigliotta $M^{2}$, Vulcano $A^{2}$, Scatozza $R^{2}$, De Angelis $R^{2}$, Selan $L^{1}$ and Ettorre $E^{2}$ \\ ${ }^{1}$ Department of Public Health and Infectious Diseases, Rome, Italy \\ ${ }^{2}$ Department of Cardiovascular, Respiratory, Nephrologic and Geriatric Sciences, Rome, Italy
}

*Corresponding author: Servello A, Department of Public Health and Infectious Diseases, Piazzale Aldo Moro, 00185, Rome, Italy, Tel: +39 0649970617; Fax +39 0649970106; E-mail: adriana.servello@uniroma1.it

Received Date: July 09, 2014, Accepted Date: September 26, 2014, Published Date: October 5, 2014

Copyright: ( 2014 , Servello A, et al., This is an open-access article distributed under the terms of the Creative Commons Attribution License, which permits unrestricted use, distribution, and reproduction in any medium, provided the original author and source are credited.

\section{Introduction}

The presence of multiple morbid conditions is the rule in older subjects [1]. The comorbidity is a major determinant of independency loss. Several pieces of evidence suggest that the overall comorbidity burden is directly associated with faster cognitive deterioration and poorer pharmacological responsiveness in Alzheimer's disease (AD) [2]. Data suggest that cardiovascular risk factors such as hypertension, hyperlipidemia, atrial fibrillation, diabetes mellitus type 2 , tobacco smoking, carotida theromasia have been found to be robust predictors of progression of $\mathrm{AD}$ [3-7], in particular when depressive disorders cooccur. Moreover, cardiovascular risk factors accelerate AD evolution. The present study was aimed to explore whether the presence of depressive symptoms and comorbidity, particularly cardiovascular risk factor, predict faster 1-year cognitive deterioration in patients with $\mathrm{AD}$, and whether such an association is independent of overall comorbidity burden. 1-year cognitive evolution was expressed as change in Clinical Dementia Rating Scale (CDR).

\section{Material and Methods}

\section{Study population}

AD patients who visited the Alzheimer Evaluation Unit of our Department, in the period between January 2011 to January 2013, were considered for this study. AD was diagnosed according to DSMIV [8] and National Institute of Neurological and Communication Disorders and Stroke and Alzheimer's Disease and Related Diseases Association (NINCDS-ADRDA) $[9,10]$ criteria. We excluded subjects with clinically relevant mood disorders, according to DSM-IV classification and those on current anti-depressant treatment, those with vascular dementia, schizophrenia, and alcohol/substance abuse, other neurological diseases, vitamin B12 deficiency. Patients with history of stroke or MRI cortical or large lacunar infarctions were also excluded.

The study was conducted according to the International Ethical Guidelines for Biomedical Research Involving Human Subjects (Declaration of Helsinki). The protocol was approved by the Human Ethics Committee from the Hospital/University "Policlinico Umberto I" of Rome. Written informed consent was obtained from each patient or his family member.

\section{Baseline measurements}

Within 3 months before inclusion, each subject underwent medical interview, physical examination, blood tests including thyroid function, vitamin B12 assay, chest X-Ray, electrocardiogram, carotid ultrasonography and brain magnetic resonance imaging. The final determination for inclusion was based on a consensus diagnosis based on clinical, functional and neuropsychological informations, laboratory test results, and MRI clinical report.At baseline, all subjects were started with acetylcholinesterase-inibithors (AChE) treatment.

Mini-mental state evaluation (MMSE), Clinical Dementia Rating Scale (CDR), Activities of Daily Living (ADL), Instrumental Activities of Daily Living (IADL) and Geriatric Depression Scale (GDS) were administered in the same day. The Cumulative Illness Rating Scale (CIRS) was administered to quantify the burden of multiple morbidity. CIRS is composed by 14 items, aimed to explore the presence of pathology in the following areas: heart, hypertension, vascular system, respiratory system, eye-ear-nose-throat, upper and lower gastrointestinal tract, hepatobiliary system, kidney, genitourinary system, musculoskeletal system, central and peripheral nervous system (not including dementia), endocrine/metabolic disorders, behavioral and psychiatric disorders. Each item is scored as follows: 1=no disease; $2=$ mild disease; $3=$ =moderate disease; $4=$ severe disease; $5=$ lifethreatening disease. Two indices are obtained from the CIRS: the severity index (CIRS SI) and the comorbidity index (CIRS CI). CIRS SI was calculated by averaging the scores of the 13 items except behavioral-psychiatric disorders; CIRS CI was calculated by counting the number of items for which a score $\geq 3$ was reported, except behavioral-psychiatric disorders $[11,12]$.

\section{One-year reassessment}

After 1 year, each patient underwent overall clinical reassessment. Mini-mental state evaluation (MMSE), Clinical Dementia Rating Scale (CDR),Activities of Daily Living (ADL), Instrumental Activities of Daily Living (IADL) and Geriatric Depression Scale (GDS) were administered.

\section{Statistical analysis}

All analyses were performed via Statistical Package for Social Sciences (SPSS), version 18.0 for Windows (SPSS Inc., Chicago, IL, USA). Continuous variables are presented as mean $\pm S D$, categorical variables as frequencies. Subjects were grouped according to CIRS-CI ( $\leq 3$ vs $\geq 4$ ). Student's t-test was used to compare baseline continuous variables, $\chi^{2}$-test for categorical variables.1-year MMSE, CDR, ADL and IADL score were compared from baseline through Student's paired t-test. Multivariable regression analysis was performed to identify the variables independently associated with higher 1-year changes in CDR. 
Citation: Servello A, Artini M, Cerra E, Vigliotta MT, Vulcano A, et al. (2014) Role of Cardiovascular Comorbidity and Depressive Symptoms on One-Year Clinical Progression of Alzheimer's Disease (AD) in a Population of Italian Elderly. J Psychiatry 17: 1000170. doi: $10.4172 / 2378-5756.1000170$

Page 2 of 4

\begin{tabular}{|l|l|l|l|}
\hline & $\begin{array}{l}\text { CIRS-CI } \\
(\mathbf{n}=\mathbf{5 2})\end{array}$ & $\mathbf{C I R S - C I ~} \mathbf{4}$ (n=50) & $\mathbf{p}$ \\
\hline Women (\%) & 73.1 & 60 & NS \\
\hline Age (years) & $80.6 \pm 8.0$ & $80.5 \pm 5.7$ & NS \\
\hline MMSE (score) & $21.2 \pm 5.5$ & $20.6 \pm 4.7$ & NS \\
\hline CDR (score) & $1.23 \pm 0.7$ & $1.32 \pm 0.6$ & NS \\
\hline GDS (score) & $3.65 \pm 3.7$ & $5.92 \pm 3.5$ & 0.002 \\
\hline ADL (score) & $5.27 \pm 1.1$ & $4.92 \pm 1.3$ & NS \\
\hline IADL (score) & $4.4 \pm 2.8$ & $3.14 \pm 2.1$ & 0.012 \\
\hline CIRS-CI (score) & $1.75 \pm 1.0$ & $5.38 \pm 1.4$ & $<0.0001$ \\
\hline CIRS-SI & $1.43 \pm 0.3$ & $2.04 \pm 0.3$ & $<0.0001$ \\
\hline Hypertension (\%) & 57.7 & 86 & $<0.0001$ \\
\hline $\begin{array}{l}\text { Atrial Fibrillation } \\
\text { (\%) }\end{array}$ & 7.7 & 12 & NS \\
\hline
\end{tabular}

\begin{tabular}{|l|l|l|l|}
\hline Diabetes (\%) & 9.6 & 28 & 0.002 \\
\hline $\begin{array}{l}\text { Carotid plaques } \\
(\%)\end{array}$ & 22 & 34 & NS \\
\hline Dyslipidemia (\%) & 36.5 & 34 & NS \\
\hline MEDICATION & & 89 & NS \\
\hline $\begin{array}{l}\text { Antihypertensive } \\
(\%)\end{array}$ & 60 & 11 & NS \\
\hline Antidiabetes (\%) & 7 & 93 & NS \\
\hline Antiplatelet (\%) & 72 & 31 & NS \\
\hline Statin (\%) & 23 & & \\
\hline
\end{tabular}

Table 1: Baseline characteristics of the sample, according to CIRS-CI score

\begin{tabular}{|l|l|l|l|}
\hline & Dependent Variable: 1-year CDR changes & & SE (B) \\
\hline Covariates & $\mathrm{B}$ & 0.011 & $\mathrm{p}$ \\
\hline Age & 0.010 & 0.143 & $\mathrm{~ns}$ \\
\hline Hypertension & -0.064 & 0.181 & $\mathrm{~ns}$ \\
\hline Atrial-fibrillation & -0.125 & 0.185 & $\mathrm{~ns}$ \\
\hline Diabetes & -0.064 & 0.164 & $\mathrm{~ns}$ \\
\hline Dyslipidemia & 0.077 & 0.162 & $\mathrm{~ns}$ \\
\hline Carotid plaques & -0.083 & 0.316 & $\mathrm{~ns}$ \\
\hline Baseline CIRS-CI & -0.004 & 0.062 & $\mathrm{~ns}$ \\
\hline Baseline CIRS-SI & 0.150 & 0.033 & $\mathrm{~ns}$ \\
\hline Baseline GDS score & 0.270 & 0.014 \\
\hline
\end{tabular}

Table 2: 1-year reassessment and differences from baseline, according to baseline CIRS-CI, ${ }^{*}$ comparison of the differences from baseline between the 2 groups. $\beta=$ regression coefficient; $\operatorname{SE}(\beta)=$ standard error of $\beta$.

Adjusted for sex, education, baseline ADL and IADL, medications and 1-year MMSE loss

\section{Results}

All subjects, baseline mean age $80.5 \pm 7.1$, achieved the 1 year elements of the study assessment. At baseline, 52 subjects achieved CIRS-CI $\leq 3,50$ subjects $\geq 4$. As shown by Table 1 , the baseline characteristics of the subjects were compared according to the CIRSCI score. No significant differences were found in sex, age, education, MMSE, CDR, ADL, prevalence of atrial fibrillation, dyslipidemia, presence of carotid plaques and medications. Subjects with CIRS-CI $\geq$ 4 exhibited significantly higher prevalence of hypertension $(\mathrm{p}<0.0001)$ and diabetes $(\mathrm{p}=0.002)$, higher GDS score $(\mathrm{p}=0.002)$ and lower IADL score $(\mathrm{p}=0.012)$. As shown by Table 2 , subjects with baseline CIRS-CI $\leq 3$ exhibited significant 1 -year worsening in $\mathrm{CDR}(\mathrm{p}<0.0001)$, ADL $(\mathrm{p}=0.011)$ and IADL $(\mathrm{p}=0.043)$ scores, but not in MMSE. CIRS-CI $\geq 4$ exhibited significant 1-year worsening in MMSE ( $\mathrm{p}=0.033)$, CDR $(\mathrm{p}<0.0001), \mathrm{ADL} \quad(\mathrm{p}=0.016)$ and IADL $(\mathrm{p}=0.043)$ scores. The worsening from baseline in MMSE $(\mathrm{p}=0.027)$ and CDR $(\mathrm{p}=0.022)$ was significantly higher in the group with baseline CIRS-CI $\geq 4$.As shown in Table 2, a multivariable regression model was constructed to explore whether cardio-metabolic risk factors and baseline GDS were independently associated with higher 1-year changes in CDR. After adjusting for sex, education, baseline ADL and IADL, medications and 1 -year MMSE loss, the baseline GDS score $(\beta=0.270 ; \mathrm{SE}=0.033$; $\mathrm{p}=0.014$ ) was found to be the only independent predictor of higher 1year changes in CDR. No associations were found for cardio-metabolic risk factors, CIRS-CI and CIRS-SI.

\section{Discussion}

The presence and severity of comorbidities strongly accelerates the progression of cognitive impairment. The burden of chronic 
Citation: Servello A, Artini M, Cerra E, Vigliotta MT, Vulcano A, et al. (2014) Role of Cardiovascular Comorbidity and Depressive Symptoms on One-Year Clinical Progression of Alzheimer's Disease (AD) in a Population of Italian Elderly. J Psychiatry 17: 1000170.

Page 3 of 4

diseasesimpairs the functional autonomy of subjects and definitely leads to frailty. Moreover the vascular contribution to cognitive impairment and dementia is globally accepted such as to recommend a Trans disciplinary, translational and transactional approach to the management of cardiovascular risk factors. As indicated by the American Heart Association/American Stroke Association "Longterm vascular risk marker interventional studies beginning as early as midlife may be required to prevent or postpone the onset of $\mathrm{VCI}$ and Alzheimer disease"[13]. The interesting finding of our study, apparently in contrast with previous reports, is that none of the cardio-metabolic risk factors were found to be associated with 1-year cognitive deterioration. Probably an observation period of 12 months is not sufficient to demonstrate the real impact of such risk factors on the progression of $\mathrm{AD}$. The study has several limitations. First, all of the enrolled subjects were on specific treatment for their cardiometabolic condition. This could certainly have a confounding role on the analysis because a good pharmacological control of cardiovascular risk factors may be able to reduce the risk of developing cognitive impairment. Furthermore, the small number of participants may limit the statistical significance of the results obtained. Hypertension is a potent risk factor for AD. The chronic exposure to hypertension leads to small vessel damage in the brain and, subsequently, cerebral hypoperfusion and hypoxaemia [14]. This leads to tissue oxidation in time that triggers neuronal degeneration. The SMART-MR study revealed that untreated hypertension is associated with cognitive decline, while there is no correlation between hypertension and hippocampal atrophy $[15,16]$. Diabetes is another pivotal risk factor for small vessel disease, and even a pre-diabetic condition, characterized by peripheral insulin resistance, is certainly involved in reducing the clearance of beta-amyloid within the brain tissue [6]. Many studies reveal that the increased risk of cognitive decline and dementia in elderly people with diabetes reflects a dual pathologic process involving both cerebrovascular damage and neurodegenerative changes [17]. Several possible pathophysiologic mechanisms, including hyperglyceamia, insulin resistance, oxidative stress, advanced glycation end products, and inflammatory cytokines, may explain the effect of glucose deregulation on dementia risk. In addition, genetic studies have found that chromosome 10 contains the genes for both late-onset $\mathrm{AD}$ and type 2 diabetes [18]. An interesting clinicopathologic study suggests that there may be a shared predisposition for developing amyloid in both the pancreas and the brain [19]. All these mechanisms may explain the accelerated progression from MCI to dementia observed in several studies [20]. In line with prior reports, we observed that subjects with higher comorbidity burden were more likely to have higher GDS scores and a lower ADL scores, confirming the associations existing between medical illness burden, mood disorders and loss of independency. The most interesting finding of our investigation is that depressive symptoms in $\mathrm{AD}$ patients are independently associated with faster cognitive deterioration in 1 year. This result, if confirmed by further large scale investigations, may indicate a role of isolated depressive symptoms on the progression of dementia, also in the absence of a clinical relevant mood disorder. Depression is the most common neuropsychiatric disorderin dementia subjects: depression occurs in $30 \%$ of $\mathrm{AD}$ patients. Most of the association between depression and $\mathrm{AD}$ remains unclear. Mood disturbances may represent the first symptom of $\mathrm{AD}$, as well as depressive disorders may be characterized by a reversible involvement of cognition. Further longitudinal investigation to better characterize the temporal nature and the direction of this association are welcome. Our results need to be confirmed on large scale, since the antidepressant treatment in $\mathrm{AD}$ patients with depressive symptoms, even not fulfilling diagnostic criteria for specific depressive disorders, may modulate the clinical evolution of dementia. Further investigations will reveal the real impact of antidepressant agents on the clinical evolution of AD. This may lead to a better management of $\mathrm{AD}$ and its related disorders, that cause severe disability of patients by rule, as well as caregiver overloading.

\section{Conflicts of Interest}

On behalf of all authors, the corresponding author states that there is no conflict of interest.

\section{References}

1. Abete P, Testa G, Della Morte D, Mazzella F, Galizia G, et al. (2004) Comorbility in the elderly: epidemiology and clinical features. Gerontol 52:267-272.

2. Doraiswamy PM, Leon J, Cummings JL, Marin D, Neumann PJ (2002) Prevalence and impact of medical comorbidity in Alzheimer's disease. J Gerontol A Biol Sci Med Sci 57: M173-177.

3. Rojo L, Sjöberg MK, Hernández P, Zambrano C, Maccioni RB (2006) Roles of cholesterol and lipids in the etiopathogenesis of Alzheimer's disease. J Biomed Biotechnol 2006: 73976.

4. Ettorre E, Cicerchia M, De Benedetto G, Fossati C, Guglielmi S, et al. (2009) A possible role of atrial fibrillation as a risk factor for dementia. Arch Gerontol Geriatr 49 Suppl 1: 71-76.

5. Shi J, Perry G, Smith MA, Friedland RP (2000) Vascular abnormalities: the insidious pathogenesis of Alzheimer's disease. Neurobiol Aging 21: 357-361.

6. Sabayan B, Foroughinia F, Mowla A, Borhanihaghighi A (2008) Role of insulin metabolism disturbances in the development of Alzheimer disease: mini review. Am J Alzheimers Dis Other Demen 23: 192-199.

7. Ettorre E, Cerra E, Marigliano B, Vigliotta M, Vulcano A, et al. (2012) Role of cardiovascular risk factors (CRF) in the patients with mild cognitive impairment (MCI). Arch Gerontol Geriatr 54: 330-332.

8. American Psychiatric Association (1994) Diagnostic and Statistical Manual of Mental Disorders, Fourth Edition. Washington, DC.

9. McKhann G, Drachman D, Folstein M, Katzman R, Price D, et al. (1984) Clinical diagnosis of Alzheimer's disease: report of the NINCDS-ADRDA Work Group under the auspices of Department of Health and Human Services Task Force on Alzheimer's Disease. Neurology 34: 939-944.

10. Dubois B, Feldman HH, Jacova C, Dekosky ST, Barberger-Gateau P, et al. (2007) Research criteria for the diagnosis of Alzheimer's disease: revising the NINCDS-ADRDA criteria. Lancet Neurol 6: 734-746.

11. de Groot V, Beckerman H, Lankhorst GJ, Bouter LM (2003) How to measure comorbidity. a critical review of available methods. J Clin Epidemiol 56: 221-229.

12. Linn BS, Linn MW, Gurel L (1968) Cumulative illness rating scale. J Am Geriatr Soc 16: 622-626.

13. Gorelick PB, Scuteri A, Black SE, Decarli C, Greenberg SM, et al. (2011) Vascular contributions to cognitive impairment and dementia: a statement for healthcare professionals from the american heart association/american stroke association. Stroke 42: 2672-2713.

14. Peters R, Beckett N (2009) Hypertension, dementia, and antihypertensive treatment: implications for the very elderly. Curr Hypertens Rep 11: 277-282.

15. Knoops AJ, van der Graaf Y, Appelman AP, Mali WP, Geerlings MI (2009) Total cerebral blood flow and hippocampal volume in patients with arterial disease. The SMART-MR study. J Cereb Blood Flow Metab 29: 1727-1733.

16. Muller M, van der Graaf Y, Visseren FL, Mali WP, Geerlings MI; SMART Study Group (2012) Hypertension and longitudinal changes in cerebral blood flow: the SMART-MR study. Ann Neurol 71: 825-833. 
Citation: Servello A, Artini M, Cerra E, Vigliotta MT, Vulcano A, et al. (2014) Role of Cardiovascular Comorbidity and Depressive Symptoms on One-Year Clinical Progression of Alzheimer's Disease (AD) in a Population of Italian Elderly. J Psychiatry 17: 1000170. doi: $10.4172 / 2378-5756.1000170$

Page 4 of 4

17. Biessels GJ, Staekenborg S, Brunner E, Brayne C, Scheltens P (2006) Risk of dementia in diabetes mellitus: a systematic review. Lancet Neurol 5 : 64-74.

18. Korf ES, White LR, Scheltens P, Launer LJ (2006) Brain aging in very old men with type 2 diabetes: the Honolulu-Asia Aging Study. Diabetes Care 29: 2268-2274.
19. Janson J, Laedtke T, Parisi JE, O'Brien P, Petersen RC, et al. (2004) Increased risk of type 2 diabetes in Alzheimer disease. Diabetes 53: 474-481.

20. Xu W, Caracciolo B, Wang HX, Winblad B, Bäckman L, et al. (2010) Accelerated progression from mild cognitive impairment to dementia in people with diabetes. Diabetes 59: 2928-2935. 